\title{
Les obstacles au dépistage du virus de I'hépatite $C$ et les facteurs qui le facilitent : examen de la portée
}

\author{
N Shehata ${ }^{1}$, T Austin $^{1 *}, \mathrm{~S} \mathrm{Ha}^{1}, \mathrm{~K} \mathrm{Timmerman}^{1}$
}

\section{Résumé}

Contexte : Dans le cadre de l'effort mondial engagé pour l'élimination du virus de l'hépatite C (VHC), il est important de comprendre quels sont les obstacles à son dépistage de même que les facteurs qui, au contraire, le facilitent.

Objectif : Examiner les obstacles au dépistage du VHC et les facteurs qui le facilitent, du point de vue des fournisseurs de soins de santé qui offrent ce service et des patients qui cherchent à l'obtenir.

Méthodologie : Une recherche documentaire a été effectuée à l'aide des bases de données Embase, Medline et Scopus afin de colliger les études publiées entre janvier 2012 et juillet 2017. Nous avons extrait les données suivantes : le nom de l'auteur, l'année de publication, le modèle des études, la population, le milieu, le pays, la méthode de collecte des données et les résultats en matière de connaissance et de sensibilisation.

Résultats : Au total, 16 articles ont été ciblés. Les obstacles au dépistage du VHC observés chez les patients comprennent une faible perception individuelle du risque de contracter le virus, la stigmatisation perçue et la peur d'un résultat positif. Les facteurs qui facilitent le dépistage du VHC et qui sont mentionnés par les patients comprennent une meilleure connaissance du mode de transmission et de la manifestation de l'infection au VHC et I'intégration du dépistage du VHC aux soins de routine, qu'il accompagne ou non le dépistage du VIH. Les obstacles au dépistage du VHC comprennent les contraintes de temps, le manque de connaissances spécifiques au VHC et la gêne de poser des questions sur les comportements à risque. Les facteurs qui facilitent le dépistage comprennent les rappels de procéder au dépistage de même que le fait de travailler dans des endroits qui présentent un nombre plus important de cas liés au VHC.

Conclusion : Le manque de connaissances comme la peur de la stigmatisation et de la discrimination demeurent des obstacles au dépistage du VHC chez les patients alors que, chez les fournisseurs de soins de santé, ce sont le manque de temps et de connaissances ainsi que la gêne de poser des questions sur les comportements à risque qui font encore obstacle au dépistage. Ces conclusions ciblent des secteurs possibles pour de futures actions dans le domaine de la santé publique.

\begin{abstract}
Affiliation
${ }^{1}$ Centre de la lutte contre les maladies transmissibles et les infections, Agence de la santé publique du Canada, Ottawa (Ontario)
\end{abstract}

Correspondance : tujuanna. austin@canada.ca

Citation proposée : Shehata N, Austin T, Ha S, Timmerman K. Les obstacles au dépistage du virus de I'hépatite $C$ et les facteurs qui le facilitent : examen de la portée. Relevé des maladies transmissibles au Canada 2018;44(7/8):186-93. https://doi.org/10.14745/ccdr.v44i78a03f

Mots-clés : obstacles, facteurs qui facilitent, dépistage, test, hépatite $C$

\section{Introduction}

En 2016, la 69 Assemblée mondiale de la santé a approuvé la Stratégie mondiale du secteur de la santé visant à éliminer d'ici 2030 le virus de l'hépatite C (VHC) et le virus de l'hépatite B (VHB) en tant que menaces à la santé publique (1). Ce but a été rendu possible grâce à la disponibilité de nouveaux traitements antiviraux à action directe (ADD) sans interférons, qui sont très efficaces pour arrêter la progression de la maladie hépatique et éliminer le virus (2).

L'un des défis posés par le diagnostic de l'infection au VHC est qu'elle est souvent asymptomatique. Le dépistage de l'infection au $\mathrm{VHC}$ est une étape fondamentale au repérage des personnes qui ignorent être porteuses de l'infection. De plus, le diagnostic d'une infection au VHC peut aider à réduire le fardeau de la maladie et à limiter sa transmission aux personnes qui présentent un risque accru d'infection et à celles qui sont à risque d'être infectées de nouveau (3). Plus le nombre de personnes infectées qui reçoivent un traitement sera grand, moins il y aura de transmission et, par le fait même, de nouveaux cas. Ainsi, le dépistage est critique à l'atteinte des cibles établies par l'Assemblée mondiale de la santé. 
En 2011, on estimait que $44 \%$ des personnes qui vivaient avec une infection chronique au VHC ignoraient qu'elles étaient porteuses de l'infection (4). Les lignes directrices canadiennes actuelles concernant le dépistage du $\mathrm{VHC}$ recommandent de procéder à un test de dépistage chez les personnes présentant un risque accru d'infection (5). Les personnes à haut risque comprennent celles qui présentent des antécédents actuels ou passés de consommation de drogues injectables, les hommes homosexuels ou bisexuels porteurs du VIH qui ont des relations sexuelles avec d'autres hommes, les populations carcérales, les personnes sans abri, les personnes qui ont été exposées à des procédures de soins de santé ou des services personnels présentant des lacunes en matière de prévention et de contrôle des infections, et les personnes nées, qui ont voyagé ou qui ont résidé dans des pays où la prévalence du VHC est élevée (6).

L'objectif de la présente recherche exploratoire de la documentation est d'examiner les obstacles actuels au dépistage du VHC de même que les facteurs qui le facilitent, du point de vue des patients comme de celui des fournisseurs de soins de santé.

\section{Méthodologie}

Dans la présente recherche exploratoire de la documentation, nous cherchons à examiner les obstacles au dépistage du $\mathrm{VHC}$ et les facteurs qui le facilitent et qui sont relevés par les fournisseurs de soins de santé, les patients et les personnes qui ne consomment pas de drogues injectables et qui ne sont pas à risque d'être porteurs d'une infection au VHC. Cette omission s'explique par le fait que les obstacles au dépistage du VHC et les facteurs qui le facilitent chez les consommateurs de drogues injectables sont bien documentés (7). De plus, les consommateurs de drogues injectables ont tendance à présenter des taux de dépistage élevés (5). Pourtant, peu d'études ont examiné les obstacles au dépistage du VHC de même que les facteurs qui le facilitent parmi les populations à risque, y compris celles qui présentent un fardeau caché.

Nous avons réalisé une recherche documentaire des études publiées entre janvier 2012 et juillet 2017 afin d'intégrer les plus récentes recherches dans le paysage changeant du dépistage du VHC. Nous avons fouillé les bases de données Embase, Medline et Scopus en utilisant les termes de recherche "hépatite $\mathrm{C}$ » ou "VHC», «dépistage» ou «test» et «obstacles» ou «qui facilite». (La stratégie de recherche est disponible sur demande)

Les études étaient retenues si elles avaient été publiées entre janvier 2012 et juillet 2017 en français ou en anglais, si elles ciblaient des populations autres que les consommateurs de drogues injectables et si elles avaient été réalisées dans des pays dotés de ressources suffisantes. Le courrier des lecteurs, les revues narratives et les commentaires ont été exclus. Les populations clés n'étaient pas prédéterminées, mais ont émergé plutôt des résultats de la recherche.

Une fois que les articles avaient été repérés, le titre et les résumés étaient triés en fonction de leur pertinence. Nous avons extrait des données portant sur la population de l'étude, le milieu où elle a été réalisée, son modèle, ses retombées et ses résultats.

\section{Résultats}

Sur les 126 articles ciblés par la recherche, 16 études portaient sur les obstacles au dépistage du VHC et sur les facteurs qui le facilitent (figure 1).

\section{Figure 1 : Organigramme de la stratégie de recherche}

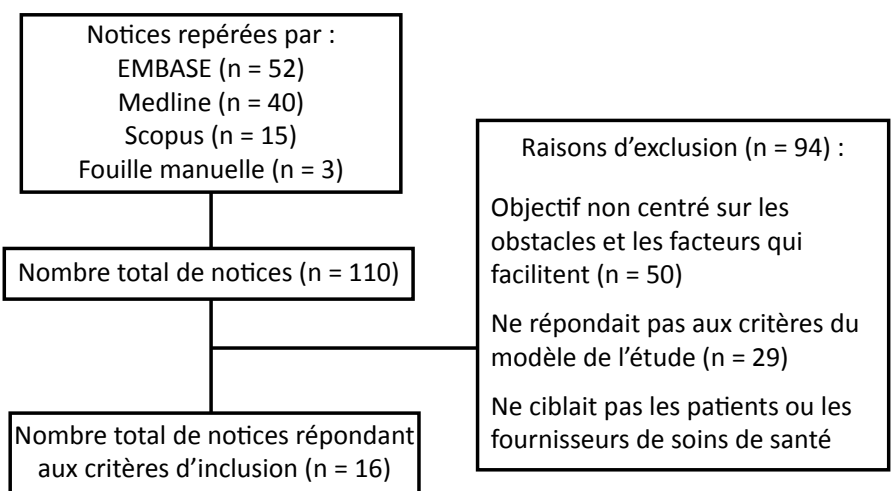

Abréviation : n, nombre

La plupart des études ont examiné à la fois la population des fournisseurs de soins de santé et celle des patients. Les fournisseurs de soins de santé comprenaient des médecins généralistes, des représentants cliniques de centres hospitaliers, des médecins récemment diplômés, des étudiants en médecine, des médecins résidents et des infirmiers (8-14). Les groupes de patients comprenaient des adultes nés entre 1945 et 1965 , des patients consultant des cliniques d'infections transmissibles sexuellement (ITS) ou des établissements de soins primaires, des adolescents et des adultes travaillant à la prévention du $\mathrm{VIH}$ et du VHC, des populations immigrantes et migrantes, ainsi que des travailleurs du sexe $(10,12,13,15-23)$.

La plupart des études ont été réalisées aux États-Unis $(n=9)$, suivis par le Canada $(n=2)$, le Royaume-Uni $(n=2)$, l'Australie $(n=1)$, la France $(n=1)$ et l'Écosse $(n=1)$. Les modèles d'étude comprenaient des études observationnelles, des études transversales et des entrevues qualitatives.

\section{Les obstacles des fournisseurs de soins de santé}

Nous avons relevé trois principales catégories d'obstacles pour les fournisseurs de soins de santé prodiguant le dépistage du VHC : les contraintes de temps, le manque de connaissances spécifiques sur le VHC et la gêne d'en discuter. Ces obstacles ont été relevés pour l'ensemble des spécialisations (c.-à-d. par les infirmiers du service des urgences, les médecins généralistes, les médecins résidents nouvellement diplômés) et des milieux (c.-à-d. les salles d'urgence, les centres de santé communautaires, les cliniques de soins primaires et d'ITS).

\section{Contraintes de temps}

Les contraintes de temps - plus spécifiquement, le temps de consultation limité, les priorités concurrentes et les longues procédures de soutien psychologique précédant et suivant le test de dépistage - étaient souvent citées comme des obstacles au dépistage du VHC $(8,10-13)$. II en résulte que les fournisseurs de soins de santé - particulièrement les médecins généralistes ne posaient pas systématiquement à leurs patients de questions 
sur les facteurs de risque du VHC $(8,21)$. On accorde souvent une basse priorité au VHC en raison de sa lente progression comparativement à d'autres problèmes de santé auxquels on doit s'attarder au cours de la consultation $(8,11,15)$.

Les longues procédures de soutien psychologique sont aussi apparues comme un obstacle aux fournisseurs de soins de santé qui offrent le dépistage du VHC. Au cours d'une étude réalisée dans un hôpital d'enseignement américain, 75 \% des patients hospitalisés n'avaient pas subi de test de dépistage, car le médecin résident avait contourné le processus de dépistage (21).

\section{Manque de connaissances spécifiques}

Les fournisseurs de soins de santé ont démontré une compréhension générale des facteurs de risque du VHC, mais ils ont reconnu le besoin d'améliorer leurs connaissances au sujet des personnes devant être testées $(8,11)$. Dans une étude réalisée en Écosse, les fournisseurs de soins de santé ont aussi reconnu avoir des connaissances limitées en ce qui a trait au soutien psychologique, aux personnes et aux endroits vers lesquels aiguiller les patients ayant obtenu un diagnostic ainsi qu'aux protocoles de dépistage (11). Le manque de connaissances portant sur les lignes directrices des tests de dépistage constituait aussi un obstacle (12).

\section{Le manque d'aisance}

La plupart des fournisseurs de soins de santé comprennent l'importance d'aborder les facteurs de risque et de dépister le VHC; cependant, plusieurs ont reconnu éprouver des difficultés à soulever le sujet avec leurs patients (9). C'était particulièrement vrai si le patient n'avait pas de contacts réguliers avec le fournisseur et si le rapport patient-fournisseur était limité. Les fournisseurs ont déclaré être plus à l'aise d'offrir le test de dépistage après avoir établi une relation avec leur patient et avoir pris connaissance de ses antécédents et de ses facteurs de risque (8).

Le milieu de pratique était un facteur important qui contribuait au sentiment d'aise des fournisseurs de soins de santé à l'égard du dépistage du VHC. Dans les régions présentant une forte prévalence de l'infection au VHC, les fournisseurs de soins de santé étaient plus à l'aise d'offrir le test de dépistage. Au contraire, les fournisseurs de soins de santé qui travaillaient dans des régions présentant une faible prévalence de l'infection au VHC étaient moins susceptibles d'être à l'aise d'offrir le test de dépistage (8).

\section{Les obstacles des patients au dépistage}

Les obstacles fréquents empêchant les patients de rechercher un dépistage du VHC comprennent un manque de connaissances, la faible perception du risque de contracter le virus, la peur, la stigmatisation et la discrimination ainsi que l'accès limité à des services de soins de santé.

\section{Le manque de connaissances spécifiques}

Le manque de connaissances sur le VHC, y compris les facteurs de risque, les voies de transmission, les manifestations et les répercussions d'une infection non traitée, ont souvent été considérés comme des obstacles empêchant les patients de rechercher un test de dépistage. Le manque de connaissances est évident pour les patients de tous les niveaux de risque. De plus, les parties prenantes en matière de VIH et de VHC croyaient que la réticence des fournisseurs de soins de santé à aborder la question du $\mathrm{VHC}$ contribuait à la réticence des adolescents et des adultes à rechercher le test de dépistage ou à en discuter (18).

\section{La faible perception du risque de contracter le virus}

Le manque de connaissances sur le $\mathrm{VHC}$ a pour résultat une faible perception individuelle du risque de contracter le virus qui, à son tour, entraîne une sous-estimation du nombre de cas non diagnostiqués d'infection au VHC. Les patients déclarent souvent ne pas être à risque de contracter une infection au VHC ou de ne présenter aucun des facteurs de risque $(11,15,19)$. D'ailleurs, les patients qui avaient subi un dépistage du VHC par le passé étaient plus susceptibles de pas chercher à en obtenir un autre à nouveau (19).

\section{La peur, la stigmatisation et la discrimination}

Dans une étude canadienne qualitative, des adolescents et des adultes agissant comme parties prenantes dans la prévention du VIH et du VHC ont affirmé que les perceptions de la stigmatisation et les attitudes négatives envers le $\mathrm{VIH}$ et le $\mathrm{VHC}$ persistent et sont un obstacle important pour les patients qui recherchent le test de dépistage (18). Selon d'autres études, des patients - particulièrement ceux issus des populations immigrantes ou migrantes - auraient peur d'un diagnostic positif du VHC, et la stigmatisation et la discrimination qui y sont associées seraient des obstacles à son dépistage $(19,20,22)$.

\section{Accès limité au système de soins de santé}

Le nombre limité d'interactions avec le système de soins de santé, le manque de fournisseurs de soins primaires et un statut socioéconomique instable ont tous été ciblés dans la littérature comme étant des obstacles au test de dépistage selon les patients. Les adultes nés entre 1945 et 1965 ont mentionné le coût du test de dépistage, l'absence de couverture $d$ 'assurance-maladie, l'accès limité aux cliniques d'hépatologie et les longs temps d'attente avant d'obtenir un rendez-vous comme étant des obstacles au dépistage du VHC $(13,19)$. Les barrières linguistiques et le manque de services de santé adaptés à la culture limitent l'accès des populations migrantes au dépistage du VHC $(20,22,23)$.

\section{Les facteurs facilitants pour les fournisseurs de soins de santé}

Deux facteurs facilitants clés pour les fournisseurs de soins de santé qui offrent le test de dépistage du VHC sont la sensibilisation et la connaissance des facteurs de risque du VHC ainsi que les rappels au moyen d'alertes intégrées au dossier médical électronique (DME).

\section{La sensibilisation et la connaissance des facteurs de risque}

La sensibilisation et la connaissance des facteurs de risque du VHC peuvent aider les fournisseurs de soins de santé à faciliter le test de dépistage. Dans une étude menée par McLeod et al. (2017) en Écosse, les médecins généralistes ont mentionné qu'ils offraient presque toujours un test de dépistage du VHC aux patients présentant des résultats d'examen de la fonction hépatique anormaux lorsqu'ils étaient sensibilisés aux facteurs de risque (11).

\section{Alertes intégrées au dossier médical électronique}

Les rappels sont des facteur qui facilitent souvent l'offre de dépistage du VHC. Ces rappels pourraient diminuer les occasions manquées de procéder au test de dépistage. Les fournisseurs 
de soins de santé ont mentionné que des alertes programmées dans le dossier médical électronique, fondées sur les antécédents de santé du patient et ses facteurs de risque, sont une méthode permettant de se rappeler de procéder au test de dépistage $(8,9)$. Cette méthode assurerait que des patients auxquels on n'a pas offert précédemment le test de dépistage ou qui l'auraient décliné par le passé ne rateraient pas d'autres occasions de procéder au dépistage. Bien qu'une étude a décrit une certaine "fatigue des alertes du dossier médical électronique» (où les fournisseurs de soins de santé reçoivent trop de rappels) (10), d'autres études ont relevé les oublis des fournisseurs de soins de santé comme étant des obstacles au dépistage et ont suggéré l'intégration d'alertes au sein du DME comme étant une solution (8).

\section{Facteurs facilitants pour les patients}

Un important facteur qui facilite la recherche d'un test de dépistage du VHC est la sensibilisation et la connaissance du VHC.

\section{La sensibilisation et la connaissance}

L'amélioration de la formation des patients apparaît comme un important facteur qui facilite le dépistage du VHC. La formation, qu'elle soit directe (c.-à-d. que les fournisseurs de soins de santé informent les patients des risques de contracter une infection au $\mathrm{VHC}$ ) ou par le moyen de campagnes de sensibilisation (p. ex., de la publicité à la télévision, des affiches ou des campagnes de santé publique), fait connaître aux patients les facteurs de risque, les voies de transmission et $d$ 'autres renseignements généraux portant sur le VHC, ce qui pourrait les rendre plus susceptibles de chercher à obtenir un test de dépistage $(8,19,20)$.

Le tableau 1 résume les principaux obstacles au dépistage du VHC ainsi que les principaux facteurs qui le facilitent. Les détails des études retenues sont présentés à l'appendice 1.

Tableau 1 : Résumé des principaux obstacles au dépistage du VHC ainsi que des principaux facteurs qui le facilitent

\begin{tabular}{|c|c|c|}
\hline Population & Obstacles & Facteurs facilitants \\
\hline $\begin{array}{l}\text { Fournisseurs } \\
\text { de soins de } \\
\text { santé }\end{array}$ & $\begin{array}{l}\text { - } \text { Contraintes de temps } \\
\text { - Manque de connaissances } \\
\text { spécifiques au VHC } \\
\text { - Gêne à discuter du VHC } \\
\text { et manque de sensibilisation } \\
\text { connaissances des } \\
\text { recommandations } \\
\text { concernant le dépistage } \\
\text { du VHC }\end{array}$ & $\begin{array}{l}\text { - Recommandation } \\
\text { concernant le } \\
\text { dépistage du VHC } \\
\text { - Alertes intégrées au } \\
\text { DME }\end{array}$ \\
\hline Patients & $\begin{array}{l}\text { - } \text { Manque de connaissances } \\
\text { - } \text { risque perception du } \\
\text { virus } \\
\text { - Crainte d'un diagnostic } \\
\text { positif } \\
\text { - Stigmatisation et } \\
\text { discrimination } \\
\text { - Barrières linguistiques } \\
\text { parmi les populations } \\
\text { migrantes } \\
\text { - Manque de services de } \\
\text { - } \text { Santé adaptés à la culture } \\
\text { - Count du dépistage } \\
\text { - Manquentialité } \\
\text { par l'assurance-maladie }\end{array}$ & $\begin{array}{l}\text { - Sensibilisation et } \\
\text { connaissance du } \\
\text { VHC } \\
\text { - Antécédents de } \\
\text { dépistage du VIH }\end{array}$ \\
\hline
\end{tabular}

Abréviations : VIH, virus de l'immunodéficience humaine; $\mathrm{VHC}$, virus de l'hépatite $\mathrm{C}$; DME, dossier médical électronique

\section{Discussion}

La présente recherche exploratoire de la documentation offre un portrait de haut niveau des obstacles et des facteurs facilitants du point de vue des fournisseurs de soins de santé qui offrent le dépistage du VHC et des patients qui veulent s'en prévaloir. Les obstacles rencontrés par les fournisseurs de soins de santé comprennent les contraintes de temps, le manque de connaissances spécifiques au VHC et la gêne d'aborder ce sujet. Les obstacles fréquents pour les patients étaient le manque de connaissances, la faible perception du risque de contracter le virus, la crainte d'un diagnostic positif, la crainte de la stigmatisation et de la discrimination, et un accès limité aux systèmes de soins de santé. Les facteurs facilitant le dépistage par les fournisseurs de soins de santé étaient les alertes intégrées au dossier médical électronique de même que la sensibilisation et la connaissance du VHC. Les facteurs facilitants permettant aux patients d'exiger un test de dépistage étaient la sensibilisation et la connaissance du VHC. Étant donnée l'estimation de la prévalence des cas non diagnostiqués à l'infection au $\mathrm{VHC}$, les résultats de la présente recherche exploratoire de la documentation sont importants afin de sensibiliser les parties prenantes aux facteurs qui facilitent ou qui entravent le dépistage.

Cet examen de la documentation présente deux limites. Premièrement, en raison de la variabilité temporelle et géographique des études, certaines font référence à des traitements antiviraux à action directe (ADD) de première génération alors que d'autres font référence à des ADD de deuxième génération. Bien que l'examen de la littérature se concentre sur les obstacles au dépistage du VHC et les facteurs qui le facilitent, il est important de prendre en compte l'éventail des traitements lorsqu'on interprète les résultats, d'autant plus que certains obstacles (c.-à-d. le coût du traitement, la crainte d'un résultat positif) peuvent être reliés à la disponibilité du traitement.

Deuxièmement, il existe certaines limites inhérentes aux modèles méthodologiques des études retenues. Par exemple, sept études parmi celles retenues suivaient un modèle transversal qui ne permet de saisir les données qu'à un moment précis dans le temps. D'autres limites, comme le biais de l'échantillon, peuvent aussi être applicables à certaines études parmi celles retenues.

Les résultats de cet examen concordent avec ceux de recherches antérieures et contribuent à la documentation portant sur le dépistage du VHC, particulièrement sur ses obstacles et les facteurs qui le facilitent. L'une des forces de cet examen est l'attention portée aux populations autres que les consommateurs de drogues injectables, telles que les autres populations à risque (personnes migrantes, travailleurs du sexe, etc.) et la population en général. De futurs travaux de recherche pourraient évaluer les obstacles au dépistage du VHC et les facteurs qui le facilitent chez des populations particulières (p. ex., les communautés autochtones, les régions éloignées ou isolées) où le diagnostic et le traitement sont prescrits dans un environnement particulier.

\section{Conclusion}

Comprendre les obstacles au dépistage du VHC et les facteurs qui le facilitent peut éclairer les plans d'action en matière de santé publique afin d'améliorer le dépistage fondé sur le risque et de réduire le nombre de personnes qui ignorent qu'elles sont porteuses de l'infection au VHC. Des patients et des fournisseurs de soins de santé mieux informés, auxquels sont donnés des moyens de faire face à la stigmatisation et au malaise entourant le sujet du VHC, permettraient de progresser vers l'éradication du virus. 


\section{Déclaration des auteurs}

N. S. - Rédaction (ébauche originale - contexte et méthodologies), conservation des données (extraction) T. A. - Conservation des données (vérification), analyse formelle, rédaction (version finale), examen et révision S. H. - Conceptualisation, méthodologie, rédaction (version finale), conservation des données, validation, analyse formelle, examen et révision, supervision, administration du projet, visualisation

K. T. - Conceptualisation, méthodologie, examen et révision, supervision, administration du projet, visualisation

$\mathrm{N}$. S. et $\mathrm{T}$. A. ont collaboré de manière équivalente à cet article.

\section{Conflit d'intérêts}

Aucun.

\section{Remerciements}

Nous voulons remercier la $D^{r e}$ Margaret Gale-Rowe, le $D^{r}$ Jun Wu et Margaret Bodie pour leurs contributions à l'examen du présent manuscrit ainsi qu'Audréanne Garand pour son soutien à la conservation des données et à l'analyse initiale.

\section{Financement}

Le présent travail a été soutenu par l'Agence de la santé publique du Canada.

\section{Références}

1. Organisation mondiale de la Santé. Stratégie mondiale du secteur de la santé contre I'hépatite virale, 2016-2021. Geneva: Organisation mondiale de la Santé; 2016. http://apps. who.int/iris/bitstream/10665/250577/1/WHO-HIV-2016.06-fre. pdf?ua $=1$

2. Micallef JM, Kaldor JM, Dore GJ. Spontaneous viral clearance following acute hepatitis $C$ infection: a systematic review of longitudinal studies. J Viral Hepat 2006 Jan;13(1):34-41. http://dx.doi.org/10.1111/j.1365-2893.2005.00651.x PubMed (https://www.ncbi.nlm.nih.gov/pubmed/16364080)

3. Westbrook RH, Dusheiko G. Natural history of hepatitis C. J Hepatol 2014 Nov;61(1 Suppl):S58-68. http://dx.doi. org/10.1016/j.jhep.2014.07.012 PubMed (https://www.ncbi. nlm.nih.gov/pubmed/25443346)

4. Trubnikov M, Yan P, Archibald C. Estimation de la prévalence de l'infection par le virus de I'hépatite $\mathrm{C}$ au Canada, 2011. Relevé des maladies transmissibles au Canada 2014 Déc;40(19):429-36. PubMed (https://www.ncbi.nlm.nih.gov/ pubmed/29769874)

5. Agence de la santé publique du Canada. Résumé des résultats clés de la phase 3 du système I-Track (2010 à 2012). Ottawa (ON): Agence de la santé publique du Canada; 2014. https:// www.canada.ca/content/dam/phac-aspc/migration/phac-aspc/ aids-sida/publication/reports/i-track-phase-3/assets/pdf/itrack-phase-3-fra.pdf

6. Pinette GD, Cox JJ, Heathcote J, Adamowski K, Riehl G. Soins primaire de l'hépatite $C$ chronique: Guide de référence professionnel 2009. Ottawa (ON): Agence de la santé publique du Canada; 2009. Le College des médecins de famille du
Canada. http://www.cfpc.ca/uploadedFiles/Resources/ Resource_Items/Health_Professionals/HEP-C-Guide_fr_2.pdf

7. Barocas JA, Brennan MB, Hull SJ, Stokes S, Fangman JJ, Westergaard RP. Barriers and facilitators of hepatitis C screening among people who inject drugs: a multi-city, mixed-methods study. Harm Reduct J 2014 Jan;11(1):1. http:// dx.doi.org/10.1186/1477-7517-11-1 PubMed (https://www. ncbi.nlm.nih.gov/pubmed/24422784)

8. Datta S, Horwood J, Hickman M, Sharp D. Case-finding for hepatitis $C$ in primary care: a mixed-methods service evaluation. Br J Gen Pract 2014 Feb;64(619):e67-74. http:// dx.doi.org/10.3399/bjgp14X677112 PubMed (https://www. ncbi.nlm.nih.gov/pubmed/24567619)

9. Duerme R, Maulana S, Schwartz J, Johnson N, Rude EJ, Laraque F. Foundation for a clinical intervention on hepatitis C: a 2015 survey of New York City hospitals' capacity to cure. Hepatology 2016;63(1):414A. https://liverlearning.aasld. org/aasld/2016/thelivermeeting/143738/fabienne.laraque. foundation.for.a.clinical.intervention.on.hepatitis.c.a.2015.html

10. Goel A, Shah B, Feuille C, Dieterich DT, Perumalswami PV. Electronic medical record flags have a limited impact on hepatitis $C$ virus birth cohort screening in the primary care setting: results of a multifaceted intervention to improve screening. Gastroenterology 2015;148(4):S123. http://dx.doi. org/10.1016/S0016-5085(15)30425-X

11. McLeod A, Cullen BL, Hutchinson SJ, Roy KM, Dillon JF, Stewart EA, Goldberg DJ. Limited impact of awareness-raising campaigns on hepatitis $C$ testing practices among general practitioners. J Viral Hepat 2017 Nov;24(11):944-54. http:// dx.doi.org/10.1111/jvh.12724 PubMed (https://www.ncbi.nlm. nih.gov/pubmed/28502088)

12. Szeto $B$, Venkat $D$, Cohen $S$. Identifying and fixing roadblocks to hepatitis $\mathrm{C}$ screening in the primary care setting. Am J Gastroenterol 2014;109:S127-8.

13. Taylor BS, Hanson JT, Veerapaneni P, Villarreal R, Fiebelkorn K, Turner BJ. Hospital-based hepatitis C screening of baby boomers in a majority Hispanic South Texas cohort: successes and barriers to implementation. Public Health Rep 2016 May-Jun;131 Suppl 2:74-83. http://dx.doi. org/10.1177/00333549161310S212 PubMed (https://www. ncbi.nlm.nih.gov/pubmed/27168665)

14. White DA, Anderson ES, Pfeil SK, Graffman SE, Trivedi TK. Differences between emergency nurse perception and patient reported experience with an ED HIV and hepatitis $C$ virus screening program. J Emerg Nurs 2016 Mar;42(2):139-45. http://dx.doi.org/10.1016/j.jen.2015.09.010 PubMed (https:// www.ncbi.nlm.nih.gov/pubmed/26547573)

15. Allison WE, Chiang W, Rubin A, Oshva L, Carmody E. Knowledge about hepatitis $C$ virus infection and acceptability of testing in the 1945-1965 birth cohort (baby boomers) presenting to a large urban emergency department: a pilot study. J Emerg Med 2016 Jun;50(6):825-831.e2. http://dx.doi. org/10.1016/j.jemermed.2016.02.001 PubMed (https://www. ncbi.nlm.nih.gov/pubmed/26954104)

16. Aparicio C, Mourez T, Simoneau G, Magnier JD, Galichon B, Plaisance P, Bergmann FJ, Sellier P. Faisabilité du dépistage ciblé VIH, VHB, VHC dans une structure de soins ambulatoires avec un système de permanence d'accès aux soins de santé intégré. Presse Med 2012 Oct;41(10):e517-23. http://dx.doi. org/10.1016/j.lpm.2012.01.039 PubMed (https://www.ncbi. nlm.nih.gov/pubmed/22464893)

17. Feldman EB, Balise R, Schiff E, Whitehead N, Thomas E. Barriers to hepatitis $C$ screening in a minority population: a 
comparison of hepatitis $C$ and human immunodeficiency virus screening rates at a community STD clinic in Miami, Florida. J Community Health 2017 Oct;42(5):921-5. http://dx.doi. org/10.1007/s10900-017-0335-6 PubMed (https://www.ncbi. nlm.nih.gov/pubmed/28353008)

18. Gahagan J, Condran B, Dube A, Jackson L, Lazarus L, Dykeman M, Harris G, Marshall Z, Proctor-Simms M, MacDonald J, Numer M. HIV/HCV prevention and youth in Atlantic Canada: implications for testing in Nova Scotia. Can J Infect Dis Med Microbiol 2015;26(Supplement B):101B. https:// www.cahr-acrv.ca/wp-content/uploads/2012/10/InfDis_26_SB_ MarApr2015_Final.pdf

19. Grannan S. Understanding patient perceptions and risk for hepatitis C screening. J Viral Hepat 2017 Aug;24(8):631-5. http://dx.doi.org/10.1111/jvh.12692 PubMed (https://www. ncbi.nlm.nih.gov/pubmed/28199776)

20. Guirgis M, Nusair F, Bu YM, Yan K, Zekry AT. Barriers faced by migrants in accessing healthcare for viral hepatitis infection. Intern Med J 2012 May;42(5):491-6. http://dx.doi. org/10.1111/j.1445-5994.2011.02647.x PubMed (https://www. ncbi.nlm.nih.gov/pubmed/22151101)

21. Patil R, Ona MA, Saikali P, Papafragkakis C, Anand S. Hepatitis $C$ screening barriers in 2016: unusual suspects. Gastroenterology 2016;150(4):S152. http://dx.doi. org/10.1016/S0016-5085(16)30610-2

22. Seedat F, Hargreaves S, Friedland JS. Engaging new migrants in infectious disease screening: a qualitative semi-structured interview study of UK migrant community health-care leads. PLoS One 2014 Oct;9(10):e108261. http://dx.doi.org/10.1371/ journal.pone.0108261 PubMed (https://www.ncbi.nlm.nih.gov/ pubmed/25330079)

23. Socías ME, Shannon K, Montaner JS, Guillemi S, Dobrer S, Nguyen P, Goldenberg S, Deering K. Gaps in the hepatitis C continuum of care among sex workers in Vancouver, British Columbia: implications for voluntary hepatitis $C$ virus testing, treatment and care. Can J Gastroenterol Hepatol 2015 Nov-Dec;29(8):411-6. http://dx.doi.org/10.1155/2015/381870 PubMed (https://www.ncbi.nlm.nih.gov/pubmed/26492129)

\section{Appendice 1 : Caractéristiques des études retenues portant sur les obstacles au dépistage du virus de l'hépatite $C$} et les facteurs qui le facilitent $(n=16)$

\begin{tabular}{|c|c|c|c|}
\hline $\begin{array}{l}\text { Auteurs(s), année de } \\
\text { publication et pays }\end{array}$ & $\begin{array}{c}\text { Modèle de l'étude, lieu et } \\
\text { population ( } n \text { ) }\end{array}$ & Obstacles & Facteurs facilitants \\
\hline $\begin{array}{l}\text { Allison et coll. (2016) } \\
\text { (15) } \\
\text { É.-U. }\end{array}$ & $\begin{array}{l}\text { Étude transversale } \\
\text { Service des urgences } \\
\text { Adultes nés entre } 1945 \text { et } 1965 \\
(n=81)\end{array}$ & $\begin{array}{l}\text { - Faible perception du risque de contracter } \\
\text { l'infection au VHC } \\
\text { - Craintes des aiguilles et refus d'analyses } \\
\text { sanguines supplémentaires } \\
\text { - L'hépatite C est moins prioritaire chez les } \\
\text { patients }\end{array}$ & $\begin{array}{l}\text { - Acceptabilité du dépistage du } \\
\text { VHC et nouvelles méthodes de } \\
\text { dépistage }\end{array}$ \\
\hline $\begin{array}{l}\text { Aparicio et coll. (2012) } \\
\text { (16) } \\
\text { France }\end{array}$ & $\begin{array}{l}\text { Étude observationnelle } \\
\text { Consultations externes } \\
\text { Adultes ayant des consultations } \\
\text { externes }(n=272)\end{array}$ & $\begin{array}{l}\text { - Les méthodes de dépistage antérieures } \\
\text { offrant des résultats négatifs étaient un } \\
\text { obstacle au dépistage }\end{array}$ & $\begin{array}{l}\text { - Intégration de renseignements } \\
\text { portant sur le dépistage dans } \\
\text { le dossier de bienvenue des } \\
\text { immigrants } \\
\text { - Médecins et infirmiers offrant } \\
\text { le dépistage aux patients se } \\
\text { présentant à l'hôpital pour tout } \\
\text { problème médical }\end{array}$ \\
\hline $\begin{array}{l}\text { Datta et coll. (2014) (8) } \\
\text { Royaume-Uni }\end{array}$ & $\begin{array}{l}\text { Étude à approches combinées } \\
\text { Six pratiques nationales de } \\
\text { services de santé à Bristol, au } \\
\text { Royaume-Uni } \\
\text { Patient à risque de contracter } \\
\text { une infection au VHC } \\
\text { ( } n=3765) \\
\text { Médecins généralistes }(n=17)\end{array}$ & $\begin{array}{l}\text { - Éprouvent des difficultés à discuter des } \\
\text { facteurs de risque avec les patients } \\
\text { - Oublient d'aborder le sujet du VHC } \\
\text { - Le VHC n'est pas une grande priorité en } \\
\text { - } \text { raison de sa lente progression } \\
\text { Priorités concurrentes (c.-à-d. temps de } \\
\text { consultation limité) } \\
\text { - Éprouvent des difficultés à établir des } \\
\text { relations avec les patients }\end{array}$ & $\begin{array}{l}\text { - Lorsque les patients divulguent } \\
\text { des facteurs de risque } \\
\text { - La sensibilisation et la } \\
\text { connaissance des facteurs de } \\
\text { risque du VHC } \\
\text { - Travailler dans un milieu } \\
\text { présentant une forte prévalence } \\
\text { du VHC }\end{array}$ \\
\hline $\begin{array}{l}\text { Duerme et coll., (2016) } \\
\text { (9) } \\
\text { É.-U. }\end{array}$ & $\begin{array}{l}\text { Étude transversale } \\
\text { Représentants cliniques } \\
(\mathrm{n}=27)^{\mathrm{a}} \\
\text { Centre hospitalier }\end{array}$ & - Capacité limitée des fournisseurs & $\begin{array}{l}\text { - Le système de DME du centre } \\
\text { hospitalier présente une alerte } \\
\text { de dépistage fondée sur l'âge } \\
\text { pour les patients à haut risque }\end{array}$ \\
\hline $\begin{array}{l}\text { Feldman et coll. (2017) } \\
\text { (17) } \\
\text { É.-U. }\end{array}$ & $\begin{array}{l}\text { Étude transversale } \\
\text { Clinique pour les ITS } \\
\text { Patients fréquentant une } \\
\text { clinique pour les ITS }(n=357)\end{array}$ & $\begin{array}{l}\text { - Les patients présentant des antécédents de } \\
\text { dépistage du VIH étaient plus susceptibles } \\
\text { d'avoir subi un test de dépistage }\end{array}$ & Non indiqué \\
\hline
\end{tabular}


Appendice 1 : Caractéristiques des études retenues portant sur les obstacles au dépistage du virus de l'hépatite $C$ et les facteurs qui le facilitent $(n=16)$ (suite)

\begin{tabular}{|c|c|c|c|}
\hline $\begin{array}{l}\text { Auteurs(s), année de } \\
\text { publication et pays }\end{array}$ & $\begin{array}{c}\text { Modèle de l'étude, lieu et } \\
\text { population }(n)\end{array}$ & Obstacles & Facteurs facilitants \\
\hline $\begin{array}{l}\text { Gahagan et coll. (2015) } \\
\text { (18) } \\
\text { Provinces de } \\
\text { l'Atlantique, Canada }\end{array}$ & $\begin{array}{l}\text { Recherche exploratoire de la } \\
\text { documentation et entrevues } \\
\text { qualitatives } \\
\text { Nova Scotia Health Research } \\
\text { Foundation } \\
\text { Parties prenantes à la } \\
\text { prévention du VIH-VHC - } \\
\text { adolescents de } 15 \text { à } 24 \text { ans et } \\
\text { adultes }(n=48)\end{array}$ & Non indiqué & $\begin{array}{l}\text { - Préjugés et attitudes négatives } \\
\text { face au VIH/VHC } \\
\text { - Le manque de compréhension } \\
\text { des fournisseurs de soins de } \\
\text { santé en ce qui concerne } \\
\text { l'importance de cibler le } \\
\text { dépistage contribue à la } \\
\text { réticence des adolescents } \\
\text { d'en discuter ou de chercher à } \\
\text { l'obtenir }\end{array}$ \\
\hline $\begin{array}{l}\text { Goel et coll. (2015) (10) } \\
\text { É.-U. }\end{array}$ & $\begin{array}{l}\text { Étude observationnelle } \\
\text { (rétrospective) } \\
\text { Milieu de soins primaires } \\
\text { Patients consultant pour des } \\
\text { soins primaires ( } \mathrm{n}=37223 \text { ) } \\
\text { Fournisseurs de soins de santé } \\
\text { non spécifiés }\end{array}$ & $\begin{array}{l}\text { - Contraintes de temps } \\
\text { - Priorités concurrentes } \\
\text { - Fatigue du DME }\end{array}$ & $\begin{array}{l}\text { - Alertes intégrées au DME afin de } \\
\text { trier les patients de la génération } \\
\text { du baby-boom } \\
\text { - Le dépistage réalisé par des } \\
\text { personnes qui ne sont pas } \\
\text { médecins (p. ex., des infirmiers) } \\
\text { pourrait être utile }\end{array}$ \\
\hline $\begin{array}{l}\text { Grannan (2017) (19) } \\
\text { É.-U. }\end{array}$ & $\begin{array}{l}\text { Étude transversale } \\
\text { Établissement de santé fédéral } \\
\text { reconnu } \\
\text { Les participants d'âge adulte } \\
\text { étaient recrutés par un } \\
\text { établissement de santé fédéral } \\
\text { reconnu }(n=111)\end{array}$ & $\begin{array}{l}\text { - Les patients ne croyaient pas être à risque ou } \\
\text { n'ont pas signalé de facteurs de risque } \\
\text { - Test de dépistage du VHC antérieur } \\
\text { - Coût du dépistage } \\
\text { - Crainte d'un résultat positif } \\
\text { - Les fournisseurs n'ont pas mentionné aux } \\
\text { patients qu'ils devaient subir un test de } \\
\text { dépistage }\end{array}$ & $\begin{array}{l}\text { - Lorsqu'on est dans la cohorte } \\
\text { de la génération du baby- } \\
\text { boom (né entre } 1945 \text { et 1965), } \\
\text { on déclenche un rappel qui } \\
\text { demande un test de dépistage. } \\
\text { - Sensibilisation et connaissance } \\
\text { des facteurs de risque du VHC }\end{array}$ \\
\hline $\begin{array}{l}\text { Guirgis et coll. (2012) } \\
(20) \\
\text { Australie }\end{array}$ & $\begin{array}{l}\text { Étude transversale } \\
\text { Établissement hospitalier } \\
\text { spécialisé en hépatologie } \\
\text { Migrants }(n=60)\end{array}$ & $\begin{array}{l}\text { - Les barrières linguistiques à l'accès aux soins } \\
\text { de santé et le fait de ne pas comprendre la } \\
\text { terminologie médicale } \\
\text { - Les barrières culturelles à l'accès aux soins de } \\
\text { - Lanté } \\
\text { - La crainte de la discrimination et de la } \\
\text { stigmatisation } \\
\text { - Le risque perçu en matière de santé } \\
\text { - Les enjeux de vie privée et de confidentialité } \\
\text { - Les soucis financiers }\end{array}$ & $\begin{array}{l}\text { - La disponibilité d'un } \\
\text { programme de dépistage (p. } \\
\text { ex., la maternité, l'immigration, } \\
\text { l'emprisonnement, les milieux } \\
\text { scolaires, les dons de sang) } \\
\text { - Une plus grande sensibilisation } \\
\text { et une meilleure formation } \\
\text { - Davantage de services de santé } \\
\text { adaptés à la culture }\end{array}$ \\
\hline $\begin{array}{l}\text { McLeod et coll. (2017) } \\
\text { (11) } \\
\text { Écosse }\end{array}$ & $\begin{array}{l}\text { Étude transversale (précédant } \\
\text { et suivant l'enquête) } \\
\text { Milieu de soins primaires } \\
\text { Des médecins nouvellement } \\
\text { diplômés et des étudiants } \\
\text { en médecine ( } n=233 \text { avant } \\
\text { l'enquête; } n=217 \text { après } \\
\text { l'enquête) }\end{array}$ & $\begin{array}{l}\text { - Des patients qui ne se considèrent pas } \\
\text { - Famme étant à risque } \\
\text { clientèle } \\
\text { - Le VHC n'est pas une priorité pour la clientèle } \\
\text { - Manque de temps pour le soutien } \\
\text { psychologique avant et après le soutien } \\
\text { psychologique } \\
\text { - Les employés possédant les compétences } \\
\text { appropriées pour le soutien psychologique } \\
\text { - } \text { Font en nombre insuffisant } \\
\text { emple sensibilisation au VHC parmi les } \\
\text { - Connaissances limitées des personnes et des } \\
\text { endroits où aiguiller les patients ayant obtenu } \\
\text { un diagnostic } \\
\text { - Connaissances limitées des protocoles de } \\
\text { dépistage et du type de personnes devant } \\
\text { être testées }\end{array}$ & $\begin{array}{l}\text { - La plupart des médecins } \\
\text { généralistes offriraient toujours, } \\
\text { ou presque toujours, le } \\
\text { dépistage du VHC aux patients } \\
\text { qui présentent des résultats } \\
\text { d'examen de la fonction } \\
\text { hépatique anormaux } \\
\text { - Sensibilisation et connaissance } \\
\text { des facteurs de risque du VHC }\end{array}$ \\
\hline $\begin{array}{l}\text { Patil et coll. (2016) (21) } \\
\text { É.-U. }\end{array}$ & 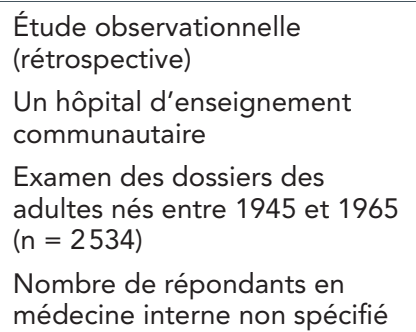 & $\begin{array}{l}\text { - Des résidents en médecine interne ont } \\
\text { contourné le test de dépistage } \\
\text { - Une date de naissance enregistrée de manière } \\
\text { erronée sur le formulaire d'admission d'un } \\
\text { résident } \\
\text { - Certains patients présentaient une altération } \\
\text { de leur état mental et ne pouvaient donner } \\
\text { leur consentement } \\
\text { - Prescription de tests de dépistage qui ne sont } \\
\text { pas envoyés au laboratoire }\end{array}$ & Non indiqué \\
\hline
\end{tabular}


Appendice 1 : Caractéristiques des études retenues portant sur les obstacles au dépistage du virus de l'hépatite C et les facteurs qui le facilitent $(n=16)$ (suite)

\begin{tabular}{|c|c|c|c|}
\hline $\begin{array}{l}\text { Auteurs(s), année de } \\
\text { publication et pays }\end{array}$ & $\begin{array}{c}\text { Modèle de l'étude, lieu et } \\
\text { population }(n)\end{array}$ & Obstacles & Facteurs facilitants \\
\hline $\begin{array}{l}\text { Seedat et coll. (2014) } \\
\text { (22) } \\
\text { Royaume-Uni }\end{array}$ & $\begin{array}{l}\text { Étude qualitative } \\
\text { Communauté migrante } \\
\text { Dirigeants des soins de santé } \\
\text { pour la communauté migrante } \\
(\mathrm{n}=20)\end{array}$ & $\begin{array}{l}\text { - Accessibilité des services de soins de santé } \\
\text { - Four les communautés migrantes } \\
\text { - Stigmate sensibilisation aux services disponibles } \\
\text { - Crainte d'un résultat positif } \\
\text { - Les services de dépistage ne sont pas adaptés } \\
\text { - Lux migrants ou à leur culture } \\
\text { - } \text { gratuits } \\
\text { - Problèmes de confidentialité }\end{array}$ & Non indiqué \\
\hline $\begin{array}{l}\text { Socias et coll. (2015) } \\
\text { (23) } \\
\text { Vancouver } \\
\text { (Colombie-Britannique) }\end{array}$ & $\begin{array}{l}\text { Étude observationnelle } \\
\text { Travailleuses du sexe }(n=705)\end{array}$ & $\begin{array}{l}\text { - Barrières linguistiques aux services de santé } \\
\text { - Participantes possédant une plus grande } \\
\text { clientèle } \\
\text { - Immigrantes vivant au Canada }\end{array}$ & Non indiqué \\
\hline $\begin{array}{l}\text { Szeto et coll. (2014) (12) } \\
\text { É.-U. }\end{array}$ & $\begin{array}{l}\text { Étude observationnelle } \\
\text { (rétrospective) } \\
\text { Milieu de soins primaires } \\
\text { Adultes nés entre } 1945 \text { et } 1965 \\
(n=395) \\
\text { Médecins résidents }(n=45)\end{array}$ & $\begin{array}{l}\text { - Manque de sensibilisation et manque } \\
\text { de connaissances des lignes directrices } \\
\text { concernant le dépistage } \\
\text { - Contraintes de temps dans les soins des } \\
\text { patients présentant des antécédents } \\
\text { médicaux complexes }\end{array}$ & Non indiqué \\
\hline $\begin{array}{l}\text { Taylor et coll. (2016) (13) } \\
\text { É.-U. }\end{array}$ & $\begin{array}{l}\text { Étude descriptive } \\
\text { Centre hospitalier } \\
\text { Adultes nés entre } 1945 \text { et } 1965 \\
(n=2327)\end{array}$ & $\begin{array}{l}\text { - Contraintes de temps (p. ex., les infirmiers } \\
\text { trouvaient le processus de consentement } \\
\text { éclairé trop pénible) } \\
\text { - Manque de couverture par l'assurance- } \\
\text { maladie chez les patients } \\
\text { - Accès limité aux cliniques d'hépatologie, } \\
\text { longues périodes d'attente pour de nouveaux } \\
\text { rendez-vous, difficultés à se retrouver dans le } \\
\text { processus de recommandation }\end{array}$ & Non indiqué \\
\hline $\begin{array}{l}\text { White et coll. (2016) (14) } \\
\text { É.-U. }\end{array}$ & $\begin{array}{l}\text { Étude transversale } \\
\text { Service des urgences } \\
\text { Infirmiers }(n=65) \text { du service } \\
\text { des urgences et patients } \\
(\mathrm{n}=491)\end{array}$ & $\begin{array}{l}\text { - Les infirmiers perçoivent souvent de façon } \\
\text { erronée la manière dont les patients vivent } \\
\text { le dépistage du VHC, en supposant qu'ils ne } \\
\text { sont pas à l'aise d'en parler }\end{array}$ & Non indiqué \\
\hline
\end{tabular}

Abréviations : DME, dossier médical électronique; ; VHC, virus de l'hépatite C; VIH, virus de l'immunodéficience humaine; ITS, infections transmissibles sexuellement; É.-U., États-Unis ${ }^{a} \mathrm{n}$ fait référence au nombre de centres hospitaliers 